\title{
Educação Permanente: Caminho para Sistematização da Assistência de Enfermagem em Saúde Mental
}

\author{
Permanent Education: Path to Systematization of Nursing Assistance in Mental Health \\ Educación Permanente: Camino hacia la Sistematización de la Asistencia de Enfermería en Salud \\ Mental
}

Simone Costa da Matta Xavier ${ }^{1}$, Elaine Antunes Cortez ${ }^{2}$

Como citar esse artigo. Xavier, SCM; Cortez, EA. Educação Permanente: Caminho para Sistematização da Assistência de Enfermagem em Saúde Mental. Revista Pró-UniverSUS. 2019 Jul./Dez.; 10 (2): 75-79.

\begin{abstract}
Resumo
Objetiva informar a pesquisa para implementar espaço permanente de reflexão-ação-reflexão sobre Sistematização da Assistência de Enfermagem com elaboração coletiva de um calendário de atividades utilizando as Metodologias Ativas, onde serão apresentados e discutidos elementos orientadores para elaboração da assistência de enfermagem e suas etapas a serem preenchidas em conjunto para (re)orientar o projeto terapêutico singular multidisciplinar. Apresenta como objetivos específicos a identificação do modelo de assistência de enfermagem existente no Centro de Atenção Psicossocial III na zona sul do Rio de Janeiro e investigar, junto a equipe de enfermagem, os limites e as possibilidades para implementação da Sistematização da Assistência de Enfermagem em Saúde Mental neste serviço. Metodologia: pesquisa qualitativa descritiva exploratória, com aplicação de questionário semi-estruturadoautoaplicado e oficinas com Metodologia do Arco de Maguerez, com os 18 profissionais de enfermagem da unidade pesquisada. Para análise será utilizada a análise de conteúdo de Bardin e a Metodologia da Problematização. Resultados: Propõe incorporar a Educação Permanente ao cotidiano para implementação da SAE em Saúde Mental, como norteador do cuidado. Considerações: Acredita ser instrumento orientador da prática cotidiana do cuidado e facilitador da comunicação entre profissionais, usuários e familiares, respeitando a transparência do SUS. E contribuir academicamente ao incorporar, com qualidade, as produções científicas a respeito e no processo de (re)construção do saber no manejo das práticas profissionais. Diminuindo a dicotomia entre o saber e fazer, além da compreensão de um novo sujeito, ativo participa continuamente da construção do processo do cuidar.
\end{abstract}

Palavras-chave: Educação Continuada em enfermagem, serviços de saúde mental, Processo de Enfermagem, Saúde Mental, Sistema Único de Saúde.

\begin{abstract}
Objectives: Objectives to inform the research to implement a permanent space of reflection-action-reflection on Nursing Care Systematization with collective elaboration of a calendar of activities using Active Methodologies, where will be presented and discussed guiding elements for the elaboration of nursing care and its steps to be taken. be completed together to (re) guide the unique multidisciplinary therapeutic project. It presents as specific objectives the identification of the nursing care model existing at the Psychosocial Care Center III in the south of Rio de Janeiro and to investigate, together with the nursing team, the limits and possibilities for implementing the Systematization of Health Nursing Care Mental in this service. Methodology: exploratory descriptive qualitative research, with the application of a semi-structured self-applied questionnaire and workshops with Maguerez Arc Methodology, with the 18 nursing professionals of the unit researched. For analysis will be used the content analysis of Bardin and the Methodology of Problematization. Results: It proposes to incorporate Permanent Education into daily life for the implementation of the SAE in Mental Health, as a guide of care. Considerations: Believes to be a guiding instrument of daily care practice and facilitator of communication between professionals, users and family members, respecting the transparency of the SUS. And contribute academically by incorporating, with quality, the scientific productions about and in the process of (re) construction of knowledge in the management of professional practices. Decreasing the dichotomy between knowing and doing, in addition to understanding a new, active subject, participates continuously in the construction of the care process.
\end{abstract}

Keywords: Continuing Education in Nursing; mental health services; Nursing Process; Mental health; Health Unic System 


\section{Resumen}

Objetivos: Su objetivo es informar a la investigación para implementar un espacio permanente de reflexión-acción-reflexión sobre la Sistematización de la Atención de Enfermería con la elaboración colectiva de un calendario de actividades utilizando Metodologías Activas, donde se presentarán y discutirán los elementos orientadores para la elaboración de la atención de enfermería y los pasos a seguir. Completarse juntos para (re) guiar el proyecto terapéutico multidisciplinario único. Presenta como objetivos específicos la identificación del modelo de atención de enfermería existente en el Centro de Atención Psicosocial III en el sur de Río de Janeiro e investigar, junto con el equipo de enfermería, los límites y las posibilidades para implementar la Sistematización de la Atención de Enfermería de Salud Mental en este servicio. Metodología: investigación cualitativa descriptiva exploratoria, con la aplicación de un cuestionario semi-estructurado auto-aplicado y talleres con Metodología MaguerezArc, con los 18 profesionales de enfermería de la unidad investigados. Para el análisis se utilizará el análisis de contenido de Bardin y la Metodología de problematización. Resultados: Propone incorporar la Educación Permanente en la vida diaria para la implementación del SAE en Salud Mental, como guía de atención. Consideraciones: cree ser un instrumento guía de la práctica de cuidado diario y un facilitador de la comunicación entre profesionales, usuarios y familias, respetando la transparencia del SUS. Y contribuir académicamente incorporando, con calidad, las producciones científicas sobre y en el proceso de (re) construcción de conocimiento en la gestión de prácticas profesionales. Disminuir la dicotomía entre saber y hacer, además de comprender un tema nuevo y activo, participa continuamente en la construcción del proceso de atención.

Palabras clave: Educación continua en enfermería; servicios de salud mental; Proceso de enfermería; Salud mental; sistema único de Salud.

\section{Introdução}

A partir da Lei $10.2016 / 2001^{1}$, propõe-se a Reforma Psiquiátrica, um novo modelo de cuidado, com a rede de atenção diária à saúde mental, norteada pelos princípios do Sistema Único de Saúde (SUS), da universalização, integralidade, equidade, descentralização e participação popular, experimenta uma importante expansão.

Os novos equipamentos substitutivos, chamados Centros de Atenção Psicossocial (CAPS), regulamentados através da Portaria $n^{\circ} 336 / 2002^{2}$, oferecem atendimento individual, em grupos, em oficinas terapêuticas, por meio de visitas domiciliares, atendimento à família e atividades comunitárias, enfatizando a integração do doente mental na comunidade e sua inserção familiar e social, atende pacientes em crise, com atendimentos diurnos (CAPS I e II), de segundafeira a sexta-feira, e em regime de 24 horas (CAPS III). Caracterizados, sobretudo, pela horizontalidade de ações interdisciplinares e terapêuticas, favorecendo a inclusão do indivíduo portador de um transtorno mental ${ }^{3}$.

Nesse olhar a Educação Permanente em Saúde (EPS) surge como estratégia para se pensar o trabalho de enfermagem, sendo "uma das formas de poder colaborar com os trabalhadores, auxiliando na compreensão das mudanças e valorizando o seu método de trabalho" "Configura-se numa estratégia fundamental para auxiliar as equipes na construção e reconstrução do cuidado ofertado aos sujeitos em sofrimento psíquico por orientar iniciativas de desenvolvimento dos profissionais e das estratégias de transformação das práticas de saúde"s.

O conceito de Educação Permanente em Saúde (EPS) definido na Política Nacional de Educação Permanente em Saúde, como "aprendizagem no trabalho, em que o aprender e o ensinar são incorporados ao cotidiano das organizações e ao processo de trabalho"6 $\mathrm{e}$ propõe que, os processos de educação dos trabalhadores da saúde se façam a partir da problematização da própria prática.

É “um dos caminhos possíveis para a interlocução entre um novo saber e o agir cotidiano(...), ação fundamental para a transformação do processo de trabalho"'.

Em 1969, em sua teoria J. Travelbee defende que para o cuidado de enfermagem é necessário que se estabeleça um processo interpessoal atendendo cinco fases: fase do encontro original, fase das identidades emergentes, fase de empatia, fase de simpatia e fase de

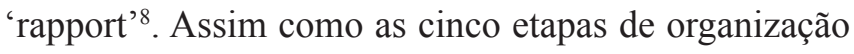
sistemática propostas no processo de enfermagem.

A partir de 1968, a Sistematização da Assistência de Enfermagem (SAE), através de seu modelo mais conhecido proposto por W. Horta (1979) começa no Brasil e constitui-se como um instrumento e metodologia para auxiliar a tomada de decisão do enfermeiro, por oferecer ordem e direcionamento ao cuidado prestado? Acredita-se que a sistematização da assistência consiste em método de trabalho para prática da enfermagem em psiquiatria e em saúde mental por ser o alcance, efetivo e concreto, das atividades de enfermagem.

O Conselho Federal de Enfermagem (COFEN), na sua resolução $\mathrm{n}^{\circ}$ 272/2002, dispõe quanto à obrigatoriedade da implementação da SAE nas Instituições de Saúde brasileiras, afirmando que deve ocorrer em todas as instituições de saúde brasileiras, públicas e privadas, considerando sua institucionalização como prática de um processo de trabalho adequado às necessidades da comunidade e como modelo assistencial a ser aplicado em todas as áreas de assistência à saúde pelo enfermeiro ${ }^{10}$.

Dessa forma o tema deste estudo se faz presente de forma bem substancial e centra-se na defesa de tese sobre a construção da Sistematização da Assistência de enfermagem em saúde mental no Centro de Atenção Psicossocial III do município do Rio de Janeiro, a luz da Educação Permanente em Saúde. Implicando assim como objeto de estudo a Educação Permanente em 
Saúde (EPS) como estratégia para a Sistematização da Assistência de Enfermagem (SAE) no Centro de Atenção Psicossocial (CAPS) III na zona Sul do município do Rio de Janeiro.

Nessa perspectiva surge como questão norteadora: De que forma a Sistematização da Assistência de Enfermagem (SAE) em saúde mental existe no Centro de Atenção Psicossocial (CAPS) III do município do Rio de Janeiro e quais os limites e possibilidades para sua implementação a partir da Educação Permanente em Saúde?

Pergunta a qual se pretende responder através do objetivo geral de implementar espaço permanente de reflexão-ação-reflexão sobre Sistematização da Assistência de Enfermagem no serviço, com elaboração coletiva de um calendário de atividades utilizando as Metodologias Ativas como produto final e por meio dos objetivos específicos de identificar o modelo de assistência de enfermagem existente no Centro de Atenção Psicossocial (CAPS) III na zona sul do Rio de Janeiro; investigar, junto a equipe de enfermagem, os limites e as possibilidades para implementação da SAE no CAPS III e propor estratégias para efetivação da Educação Permanente nesse espaço com vistas a SAE em Saúde Mental.

Dessa forma propondo um espaço onde serão apresentados e discutidos elementos orientadores para elaboração da assistência de enfermagem baseada na teoria de Joyce Travelbee e suas etapas a serem preenchidas em conjunto pela equipe de enfermagem, o paciente e a família para (re)orientar o projeto terapêutico singular multidisciplinar.

\section{Materiais e método}

Trata-se de uma pesquisa qualitativa, por permitir compreender o problema a partir da perspectiva dos sujeitos que o vivenciam. De caráter descritivo exploratório em virtude dos objetivos que se apresentaram e tendo como proposta um produto a ser construído ${ }^{11}$.

Com aplicação do modelo pesquisa convergente assistencial visto que se propõe a investigar fenômenos ocorridos no espaço da convergência das atividades de investigação com as atividades de prática assistencial ${ }^{12}$. Pretende-se aplicação de questionário semi-estruturado ${ }^{13}$ e realização de oficinas, no campo de trabalho.

Se realizará em centro de atenção psicossocial III, localizado na zona sul do município do rio de janeiro, que possui horário de atividades 24 horas por dia, durante todos os dias da semana, desde 2012, com oferta de oito leitos de crise.

A pesquisa está inserida no Programa de Pósgraduação Mestrado Profissional Ensino em Saúde (MPES/UFF) e será submetida aos comitês de ética em pesquisa (CEP) do Hospital Universitário Antônio Pedro (HUAP/UFF) e da Secretaria Municipal de Saúde do Rio de Janeiro (SMS-RJ), via Plataforma Brasil, e encaminhado à unidade a ser pesquisada, para anuência direta.

Assegura-se a preservação à identidade dos participantes através da identificação por códigos de letras do alfabeto português números arábicos, proteção dos dados fornecidos através de arquivo digital criptografado e arquivado em computador pessoal da pesquisadora e não obrigatoriedade em participar da pesquisa, porém garante que será de extrema importância para os campos de formação e pessoal, deixando claro que, a qualquer momento, será possível desistir de participar e retirar seu consentimento, sem que haja prejuízo em sua relação com o pesquisador ou com a unidade de Saúde.

Informamos estar atentos para necessidades de assistência integral decorrentes de eventuais danos, físico, psíquico, moral, intelectual, social, cultural ou espiritual, mesmo que mínimos, gerados a partir da pesquisa. E que poderá haver um risco mínimo de se constranger, de cansaço ou aborrecimento ao responder alguma pergunta durante as etapas da pesquisa. Caso aconteça, esse desconforto deverá ser minimizado pelo pesquisador responsável ou orientador.

É garantido acesso aos resultados e que sempre serão respeitados os valores culturais, sociais, morais, religiosos e éticos. Esclarece que não acarretará nenhum ganho financeiro ao pesquisador, aos participantes da pesquisa nem a instituição pesquisada, bem como não haver necessidade de treinamentos ou cursos prévios para participação na pesquisa, apenas cumprir com os critérios de inclusão e exclusão pré estabelecidos pela pesquisadora e assinatura do Termo de Consentimento Livre e Esclarecido.

Atualmente a instituição conta como equipe de enfermagem (enfermeiros, técnicos de enfermagem e auxiliares de enfermagem), um total de 18 profissionais, sendo 6 (sete) enfermeiros (as) e 11 (onze) técnicos (as) de enfermagem em regime de plantões de 24 horas semanais e 1 (um) enfermeiro(a) diarista, distribuídos em atividades assistenciais.

Serão, em sua totalidade, convidados a participação, visando a preservação e adesão dos demais participantes exclui-se o enfermeiro em atividade de coordenação técnica e os profissionais que estiverem afastados por motivos de férias, licenças médicas ou viagens durante o período de coleta de dados no ano de 2019.

Todo o material, desde locomoção, aplicação e redação, será custeado e fornecido pela pesquisadora, ocorrerá durante horário de atividade laboral dos participantes, em dia das reuniões de equipe, previamente agendados, visando respeitar os preceitos da educação permanente e para que não haja quaisquer custos para 
os profissionais da unidade.

$\mathrm{Na}$ tentativa de conhecer as percepções e caracterizar os participantes foi elaborado, junto a orientadora, um questionário semi-estruturado, apresentado a todos os participantes que aceitarem compor o corpus da pesquisa, que respeitasse o tamanho de amostra; atentasse para a redação das questões; a forma de análise dos dados; margem de erro e o processo de seleção dos participantes, a fim de complementar e enriquecer a discussão e reflexão do tema abordado.

Serão realizadas oficinas, através do uso das cinco etapas do Arco de Maguerez: observação da realidade e definição do problema, pontos-chave, teorização, hipóteses de solução e aplicação à realidade ${ }^{14}$.

Definido o problema a investigar, inicia-se uma reflexão acerca dos possíveis fatores e determinantes relacionados ao problema, possibilitando uma maior compreensão da complexidade e da multideterminação do mesmo. Tal reflexão culminará na definição dos Pontos-chave do estudo.

A Teorização, momento seguinte, é onde os dados obtidos, registrados e tratados, são analisados e discutidos, buscando-se um sentido para eles. Então se chega à etapa das Hipóteses de Solução, em que a criatividade e a originalidade devem ser bastante estimuladas para se pensar nas alternativas de solução.

Por fim, a última etapa é da Aplicação à Realidade que possibilita o intervir, o exercitar, o manejar situações associadas à solução do problema, e contempla o comprometimento do pesquisador para voltar para a mesma realidade, transformando-a em algum grau.

Para estabelecer os pontos-chave, há a identificação de alguns possíveis fatores associados ao problema, como o que estaria ocasionando a existência do problema, de forma mais direta, naquele recorte de realidade, são, em geral, aspectos contextuais como políticos, econômicos, éticos, filosóficos, envolvendo valores. etc.

$\mathrm{Na}$ quarta etapa, há construção de hipóteses de soluções realistas, conforme a urgência, para atingir diretamente os problemas levantados da realidade, os autores destacam que é o momento de criatividade. Assim caminhamos para a etapa prática onde os participantes, visando sua transformação e contribuindo para "proporcionar o desenvolvimento do compromisso social", se percebem "no processo como agentes ativos de sua transformação"14.

Para desenvolvimento dessas etapas os grupos serão estimulados por dinâmica de sensibilização e conduzidos a debater questões apresentadas no questionário semi-estruturado e a sintetizar as respostas para construção do Mapa Conceitual.

Esclarecemos ainda que todos os dados serão registrados em equipamento de áudio e diário de campo da pesquisadora para posterior transcrição e análise.

Análise dos dados se dará através da análise do conteúdo de Bardin (1977) por se considerar um conjunto de técnicas de análise das comunicações, que utiliza procedimentos sistemáticos e objetivos de descrição do conteúdo das mensagens. ${ }^{15}$

No segundo momento as oficinas serão analisadas segundo a Metodologia da problematização ${ }^{16}$. Sua se deu a partir da busca pela consonância com a PNEPS, visto que sugere problematizar as práticas, identificando primeiramente o problema, ampliando o conhecimento, desenvolvendo competências específicas e da equipe para, então, buscar soluções, colocá-las em prática e avaliá-las.

\section{Resultados Esperados}

Pretende-se discutir os dados, levantados durante a pesquisa, a luz da Política Nacional de Educação Permanente em Saúde (PNEPS) e a partir dos referenciais teórico de Donald Schon e de enfermagem de Joyce Travelbee.

Ao propor incorporar a EPS no cotidiano da equipe de enfermagem, acreditamos que assim seja norteador da construção do cuidado e instrumento orientador da prática cotidiana do cuidado de enfermagem e como facilitador da comunicação entre profissionais, usuários e familiares, respeitando assim assim a política de transparência pressuposta no SUS.

Espera-se que este estudo contribua na incorporação do processo de (re)construção do saber a esta organização de trabalho e no manejo das práticas profissionais. Contribuir com a comunidade acadêmica ao incorporar este estudo, com qualidade, as produções científicas a respeito da temática. Permitindo o aprofundamento da proposta do Ministério da Saúde relacionado à Educação Permanente em Saúde, a diminuição da dicotomia entre o saber e o saber fazer, além da compreensão de um novo sujeito/ paciente, ativo que através da lógica da participação popular, participa continuamente da construção do processo do cuidar.

\section{Referências}

1. Brasil. Lei no 10.216. Dispõe sobre a proteção e os direitos das pessoas portadoras de transtornos mentais e redireciona o modelo assistencial em saúde mental. Brasília,(6 de Abril de 2001).

2. Brasil. Portaria MS/GM n ${ }^{\circ}$ 336. Define e estabelece diretrizes para o funcionamento dos Centros de Atenção Psicossocial. Brasília, DF, (19 de fevereiro de 2002)

3. Machado VC, Santos MAFamily support from the perspective of patients in psychiatric rehospitalization: a qualitative study. Interface - Comunic.,Saude, Educ., 16(42) 793-806, 2012.

4. Brasil, Ministério da Saúde. Política Nacional de Educação Permanente em Saúde. Série B. Textos Básicos de Saúde) (Série Pactos pela Saúde 2006; v. 9)-Brasília: Ministério da Saúde, 2009. 64 p.

5. Costa TD, Gonçalves LC, Cortez EA; Tavares CM, Peixoto LS, et al. Persp. online: biol. \& saúde, Campos dos Goytaczses, 23(7), 9-15,2017. 
6. Portaria $\mathrm{MS} / \mathrm{GM} \mathrm{n}^{\circ}$ 1.996. . Dispõe sobre as diretrizes para a implementação da Política Nacional de Educação Permanente em Saúde. Diário Oficial da União, Poder Executivo, Brasília, DF (20 de Agosto de 2007).

7. Rezende R, Oliveira JEE, Friestono JKO. A educação permanente em enfermagem e o uso das tecnologias: uma revisão integrativa. R. Interd. 10(1), 190-199, 2017

8. Vasconcelos C, Boaventura P, Lima L, Volpe C, Funguetto S, Stival M. Nurses' knowledge about systematization of nursing assistance. RevEnferm UFPE online [Internet]. Recife (PE), 5, dez 2010.

9. Toledo VP. Sistematização da assistência de enfermagem psiquiátrica em um serviço de reabilitação psicossocial. [tese doutorado]. Escola de Enfermagem de Ribeirão Preto, Universidade de São Paulo, Ribeirão Preto. 2004.

10. Resolução $\mathrm{n}^{\circ}$ 272. Normatiza a Sistematização da Assistência de Enfermagem como modelo assistencial privativo do enfermeiro. Rio de Janeiro (RJ); 2002.

11. Gil AC. Como Elaborar Projetos de Pesquisa. 5. Ed. São Paulo: Atlas, 2010 .

12. Trentini M. Pesquisa Convergente Assistencial-PCA: delineamento provocador de mudanças nas práticas de saúde. Porto Alegre: Moriá, 176, 2014.

13. Bruggeman MS. Sistematização da Assistência de Enfermagem: Construção coletiva para implantação em hospital psiquiátrico [dissertação de mestrado]. Florianópolis, SC. 192. 2015.

14. Colombo AA, Berbel NAN. Metodologia da Problematização com o Arco de Maguerez e sua relação com os saberes de professores. Semina: Ciências Sociais e Humanas, Londrina, 28(2), 121-146, 2007.

15. Silva LAA, Schmidt SMS, Noal HC, Signor E, Gomes IEM. Avaliação da Educação Permanente no Processo de trabalho em Saude. Rev. Trab. Educ. Saúde, Rio de Janeiro, 14(3), 765-781. 2016.

16. Melo MC, Queluci GC, Gouvea MV. Problematizing the multidisciplinary residency in oncology: a practical teaching protocol from the perspective of nurse residents. Revista da Escola de Enfermagem da. USP, São Paulo 48(4),706-714, 2014. 